\title{
Intervenção fisioterapêutica com suporte parcial de peso em indivíduos pós-ave: revisão sistemática
}

Objetivo: Revisar sistematicamente a eficácia do suporte parcial de peso no paciente hemiparético após Acidente Vascular Encefálico. Materia e Métodos: Realizou-se levantamento de bibliografia nas bases de dados PubMed, Scielo, Science Direct e Bireme, publicadas a partir de 1998 até 2010, no qual os onze itens descritos na Escala PEDro foram utilizados como critérios de classificação dos artigos. Essa pesquisa foi realizada em maio e perdurou até setembro de 2010. Resultados: Houve evidência significativa na melhora da resistência, do equilíbrio, da evolução, da habilidade e da velocidade da marcha dos pacientes hemiparéticos pós-AVE subagudo e crônico. Conclusão: A utilização dos programas de treino de marcha com o suporte parcial de peso corporal mostrou-se eficaz em boa parte dos artigos avaliados.

Palavras-chave: Suspensão de Peso Corporal; Derrame; Esteira; Reabilitação.

\section{Physiotherapeutic intervention with partial body-weight support in subjects post stroke: systematic review}

Objective: Review sistematically the efficacy of the partial body weight support in hemiparetic subjects after stroke. Material and Methods: A survey of bibliography was conducted in the databases PubMed, Scielo, Science Direct and Bireme, published since 1998 until 2010, using the eleven items described in PEDro Scale as classification criteria. This search was held between May and September 2010. Results: There was significant evidence of the improvement in endurance, balance, evolution, ability and gait speed of hemiparetics subjects after subacute and chronic stroke. Conclusion: The use gait training programs with body weight support showed efficacy in most of the reviewed articles.

Keywords: Body Weight Suspension; Stroke; Treadmill; Rehabilitation.

Topic: Fisioterapia

Reviewed anonymously in the process of blind peer.
Received: 08/01/2017

Approved: 24/06/2017
Diogo Costa Garção

Universidade Federal de São Carlos, Brasil http://lattes.cnpq.br/8860280866778460 diogoufscar@yahoo.com.br

Juliana Martins Sobral

Universidade Federal de Sergipe, Brasil http://lattes.cnpq.br/0401429374020509 zuzusobral@hotmail.com

Júlio Cezar Dantas Santos Universidade Federal de Sergipe, Brasil http://lattes.cnpq.br/8916847349602124 julioc dantas@hotmail.com
Vanessa Brenha Duailibe Mendonça Universidade Federal de Sergipe, Brasil http://lattes.cnpq.br/7104159409018476 vanduailibe@gmail.com

\section{Lucas Silva Rezende}

Universidade Federal de Sergipe, Brasil http://lattes.cnpq.br/0241728613302636 lucas silva rezende@hotmail.com

Mylena Maria Salgueiro Santana Universidade Federal de Sergipe, Brasil http://lattes.cnpq.br/7187109306150949 ftmylenasantana@gmail.com
Patrícia Almeida Fontes

Universidade Tiradentes, Brasil

http://lattes.cnpq.br/8237336200028543 fontesfisio@yahoo.com.br

Tássia Virginia de Carvalho Oliveira Universidade Tiradentes, Brasil http://lattes.cnpq.br/2273361044731342 tassinhafisio@yahoo.com.br
Referencing this:

GARÇÃO, D. C.; SOBRASL, J. M.; SANTOS, J. C. D.; MENDONÇA, V. B. D.; REZENDE, L. S.; SANTANA, M. M. S.; FONTES, P. A.; OLIVEIRA, T. V. C.. Intervenção fisioterapêutica com suporte parcial de peso em indivíduos pós-ave: revisão sistemática. Scire Salutis, v.7, n.1, p.6-14, 2017. DOI: http://doi.org/10.6008/SPC2236-9600.2017.001.0001 


\section{INTRODUÇÃO}

Com o progresso na ciência, houve melhorias na saúde, na qualidade de vida, o que contribui para uma população com a expectativa de vida mais alta. A partir disto aumenta-se, concomitantemente, o índice de patologias crônicas características dessa população, dentre elas o Acidente Vascular Encefálico (AVE) (MCCAIN et al., 2008).

O AVE é a doença cardiovascular que mais acomete o Sistema Nervoso Central, bem como, a principal causa de incapacidades físicas e cognitivas em adultos. Suas causas estão relacionadas com a interrupção do fluxo sanguíneo para o encéfalo, originado tanto por obstrução de uma artéria que o supre (isquêmico), quanto por ruptura de um vaso (hemorrágico) (FUGL-MEYER et al., 1975). O AVE isquêmico caracteriza-se clinicamente por ser uma doença de instalação rápida, com distúrbios da função global ou focal do cérebro e uma de suas causas é a diminuição do fluxo sanguíneo cerebral (SABARI et al., 2005). O AVE hemorrágico é, em geral, mais grave com freqüência, leva a diminuição do nível de consciência ou até mesmo o coma. Em ambos, dependendo do local da lesão, podem ocorrer distúrbios da fala, visão, deglutição e marcha. Estatisticamente, $70 \%$ dos casos são isquêmicos e 30\% são hemorrágicos (LINCOLN e LEADBITTER, 1979).

As consequências funcionais dos déficits neurológicos primários continuamente predispõem os pacientes a um estilo de vida sedentário, o que limita as atividades de vida diárias (AVD's), aumenta o risco de quedas, reduz as reservas cardiovasculares, possivelmente contribui para aumento do risco de AVE recorrente e patologias cardiovasculares. Mesmo aqueles pacientes que alcançam independência funcional após o dano, constatou-se que o prejuízo motor, a longo prazo, pode constranger a vida e seu sustento (CUNHA et al., 2002; DAVID et al., 2006; HARVEY, 2003). Os pacientes frequentemente têm diminuição do equilíbrio ou força na extremidade inferior requisitada para assumir a ortostase e sustentar seus pesos nos membros inferiores (BARBEAU et al., 1993; BURGESS et al., 2010; VISINTIN et al., 1998).

Aproximadamente $2 / 3$ dos pacientes agudos internados não conseguem deambular independentemente. Embora muitos recuperem parte da deambulação, suas distâncias de caminhada estão reduzidas pela espasticidade, equilíbrio pobre, diminuição da flexão plantar e do controle motor da extremidade inferior afetada, baixa velocidade e resistência limitada, fraqueza motora residual, sinergias de movimento anormais (CHENA et al., 2005; CUNHA et al., 2002; DAVID et al., 2006). Alterações biomecânicas da marcha trazem características como passadas e comprimento de passos reduzidos, aumento dos períodos de apoio, alteração dos períodos da fase de balanço e base de suporte alargada. Hiperextensão de joelho durante a fase de apoio no membro afetado é comum, bem como a falta de controle da dorsiflexão do tornozelo. A lentidão, assimetria e o padrão ineficiente da marcha normalmente limitam a habilidade de realização de atividades de vida diária (FRANCESCHINI et al., 2009). Por 3 meses os sobreviventes do AVE ainda são incapazes de andar mesmo com assistência dependência da cadeira de rodas (KOSAK et al., 2000).

A recuperação da marcha nesses pacientes aconteceria pelo seu treinamento repetitivo, fazendo com que o cérebro realize novas sinapses e estas se sobreponham às da área afetada (WALKER et al., 2010). Para tanto são requeridas diferentes técnicas, porém, a demanda de assistência do terapeuta para ajudar o 
paciente a sustentar o peso corporal e o controle da postura é considerável (CUNHA et al., 2002; KOSAK et al., 2000). Devido ao grande número de pacientes que sofreram um AVE e a necessidade de tratamento com maior sucesso se torna mais fácil entender o porquê de numerosos subsídios terem sido inventados, desenvolvidos e recomendados ao longo dos anos, dentre eles o Suporte Parcial de Peso (SPP) (DAVIES, 1999).

O SPP é uma técnica que oferece ao paciente, durante o tratamento, a remoção de peso simétrico das extremidades inferiores, o que facilita a caminhada (VISINTIN et al., 1998). O uso de SPP como treinamento de marcha em pacientes com afeç̧ões neurológicas é feito através de uma esteira rolante conectada a fios suspensos que suportam mecanicamente o paciente durante a caminhada, juntamente com a fixação do cinturão pélvico no paciente para descarregar uma porcentagem de peso corporal específica e determinada pelo terapeuta, bem como sua velocidade (LINCOLN e LEADBITTER, 1979; MAGAGNIN et al., 2010). A pelve é anexada a uma espécie de "robô" móvel, que faz com que a base do sistema se adeque de acordo com as forças detectadas pelo robô, funciona através de um sistema de pilhas localizado no cinturão pélvico que é colocado no paciente, promovendo assim segurança e estabilidade (BURGESS et al., 2010).

Conhecer essa nova técnica de tratamento em pacientes com uma patologia neurológica como o AVE é o propósito deste estudo, contribuindo para a discussão de aspectos relacionados à nossa realidade. 0 objetivo desta revisão foi avaliar se o uso do SPP é eficaz no tratamento do paciente hemiparético pós-AVE.

\section{METODOLOGIA}

Utilizou-se algumas bases de dados eletrônicas (PubMed, Scielo, Science Direct, Bireme) para a procura dos estudos a serem incluídos, publicados desde 1998 à 2010, sendo a língua inglesa o idioma utilizado.A pesquisa foi realizada no período de maio à setembro de 2010. As seguintes palavras-chave foram utilizadas: body weight suspension, stroke, treadmill, rehabilitation (CHENA et al., 2005).

Foram selecionados os artigos com escore maior que cinco fazendo-se uso da escala PEDro, a qual tem o objetivo de identificar quais estudos clínicos poderão ter validade interna, externa (aplicabilidade do estudo) e informação estatística para que os resultados possam ser interpretados.

Os critérios são: (a) Os critérios de elegibilidade foram especificados; (b) Os sujeitos foram aleatoriamente distribuídos por grupos; (c) A distribuição dos sujeitos foi cega; (d) Inicialmente, os grupos eram semelhantes no que diz respeito aos indicadores de prognóstico mais importante; (e) Todos os sujeitos participaram de forma cega do estudo; (f) Todos os fisioterapeutas que administraram a terapia fizeram-no de forma cega; (g) Todos os avaliadores que mediram pelo menos um resultado-chave fizeram-no de forma cega; (h) Medições de pelo menos um resultado-chave foram obtidas em mais de $85 \%$ dos sujeitos inicialmente distribuídos; (i) Todos os sujeitos a partir dos quais se apresentaram medições de resultados receberam o tratamento ou a condição de controle conforme a distribuição ou, quando não, fez-se análise dos dados para pelo menos um dos resultados-chave por "intenção de tratamento"; (j) Os resultados das comparações estatísticas intergrupos foram descritos para pelo menos um resultado-chave; $(\mathrm{k}) \mathrm{O}$ estudo apresenta tanto medidas de precisão como medidas de variabilidade para pelo menos um resultado-chave. 
Além da escala PEDro, foram utilizados os seguintes critérios de inclusão: população alvo de pacientes pós-AVE, levando em consideração as fases subagudas e crônicas determinadas pelos respectivos artigos, sendo que as intervenções utilizadas foram suporte parcial de peso e tratamento fisioterapêutico convencional.

\section{RESULTADOS}

Foram encontrados quarenta e seis artigos que possuíam os critérios de inclusão. Destes, apenas nove alcançaram escore cinco na escala PEDro, os quais foram analisados quanto à eficácia do SPP (BARBEAU e VISINTIN, 2003; BURGESS et al., 2010; DANIELSSON e SUNNERHAGEN, 2000; FRANCESCHINI et al., 2009; PEURALA et al., 2005; SULLIVAN et al., 2002; VISINTIN et al., 1998; YAGURA et al., 2006; YANG et al., 2010).

Em sete dos artigos avaliados houve aumento significativo no equilíbrio, no treino de marcha, na resistência, na velocidade, no mapa cortical, diminuição do limiar motor e na demanda de oxigênio (BARBEAU e VISINTIN, 2003; BURGESS et al., 2010; DANIELSSON e SUNNERHAGEN, 2000; PEURALA et al., 2005; SULLIVAN et al., 2002; VISINTIN et al., 1998; YANG et al., 2010). Em um artigo não foi encontrado resultado significativo, provavelmente devido ao fato de que o dano nas vias motoras descendentes e os gânglios da base que causam severa hemiparesia podem ter diminuído o efeito da terapia facilitatória nos pacientes (YAGURA et al., 2006). Em outro, o fato do tratamento dos pacientes do grupo experimental ser de maneira deficiente, pode ter mascarado a presença de mudanças irrelevantes (FRANCESCHINI et al., 2009). Totalizando dois artigos, nos quais não houve resultados significativos com o uso do SPP.

Vale ressaltar que em todos os artigos um número relativamente grande de variáveis foi encontrado, tais como o tempo de tratamento (15 a 40 minutos), a quantidade de sessões ( 1 a 20), a porcentagem de peso corporal utilizada (30\% a 50\%), o uso de fisioterapia convencional ou não. Isso dificulta a padronização dos resultados, sugerindo-se que o ideal seria ter um direcionamento nos protocolos de tratamento para os próximos estudos sobre o SPP. Em alguns deles, vimos que se usa fisioterapia motora concomitante ao treino de marcha de SPP (PEURALA et al., 2005; YAGURA et al., 2006; YANG et al., 2010).

Com relação à quantidade de sessões, não houve uma homogeneidade entre os artigos. Barbeau e Visintin (2003) obtiveram resultados com 24 sessões; Yang et al. (2010) com 12 sessões; Peurala et al. (2005) com 15 sessões; Sullivan et al. (2002) com 12 sessões. Por fim, Franceschine et al. (2009) e Yagura et al. (2006) não obtiveram resultados numa média de 18 sessões. No Quadro 1 são dadas algumas características dos estudos avaliados.

Quadro 1: Características dos estudos avaliados.

\begin{tabular}{|l|l|l|}
\hline \multicolumn{1}{|c|}{ Autores } & \multicolumn{1}{|c|}{ Intervenção } & \multicolumn{1}{c|}{ Grupos de comparação } \\
\hline $\begin{array}{l}\text { Danielsson A, Sunnerhagen KS (ano de } \\
\text { publicação: 2000) }\end{array}$ & $\begin{array}{l}1 \text { sessão/semana } \\
2 \text { semanas } \\
\text { Barbeau H e Visintin M (ano de }\end{array}$ & $\begin{array}{l}\text { Grupo Experimental (9 indivíduos) } \\
\text { Grupo Controle (9 indivíduos) }\end{array}$ \\
publicação: 2003) & 4 sessões/semana & \\
& 6 semanas & Grupo experimental (com SPP) \\
& 20 minutos & Grupo controle (sem SPP) \\
& $40 \%$ de SPP progressivamente & \\
& diminuindo & \\
\hline
\end{tabular}




\begin{tabular}{|c|c|c|}
\hline $\begin{array}{l}\text { Visintin M, Barbeau H, Bitensky NK, } \\
\text { Mayo NE } \\
\text { (ano de publicação; 1998) }\end{array}$ & $\begin{array}{l}4 \text { sessões/semana } \\
6 \text { semanas } \\
40 \% \text { de SPP progressivamente } \\
\text { diminuindo }\end{array}$ & $\begin{array}{l}\text { Grupo experimental - com SPP ( } 29 \\
\text { pessoas) } \\
\text { Grupo controle - sem SPP ( } 23 \text { pessoas) }\end{array}$ \\
\hline $\begin{array}{l}\text { Burgess JK, Weibel GC, Brown DA } \\
\text { (ano de publicação: 2009) }\end{array}$ & Não foi especificado & $\begin{array}{l}\text { De } 40 \text { a } 72 \text { anos ( } 11 \text { pessoas) } \\
\text { De } 27 \text { a } 68 \text { anos ( } 12 \text { pessoas) }\end{array}$ \\
\hline $\begin{array}{l}\text { Yang YR, Chen IH, BS, Liao KK, Huang CC, } \\
\text { Wang RY (ano de publicação: 2010) }\end{array}$ & $\begin{array}{l}\text { SPP + exercício: } \\
12 \text { sessões de } 30 \text { minutos cada } \\
4 \text { semanas } \\
\text { Exercícios gerais por } 20 \text { minutos } \\
\text { Controle: } \\
12 \text { sessões de } 50 \text { minutos } \\
4 \text { semanas } \\
\text { Menos de } 40 \% \text { de SPP diminuindo } \\
\text { progressivamente }\end{array}$ & $\begin{array}{l}\text { Experimental: } \\
\text {-Agudo (até } 6 \text { meses) } \\
\text {-Crônico (mais de } 12 \text { meses) } \\
\text { Controle: } \\
\text {-Agudo (até } 6 \text { meses) } \\
\text {-Crônico (mais de } 12 \text { meses) }\end{array}$ \\
\hline $\begin{array}{l}\text { Peurala SH, Tarkka IM, Pitkänen K, } \\
\text { Sivenius J (ano de publicação: 2005) }\end{array}$ & $\begin{array}{l}3 \text { semanas } \\
5 \text { sessões de } 20 \text { minutos cada } \\
\text { Fisioterapia adicional de } 55 \text { minutos } \\
\text { Menos de } 20 \% \text { de SPP }\end{array}$ & $\begin{array}{l}\text { Treino de marcha com eletro } \\
\text { estimulação funcional (FES) } \\
\text { Treino de marcha sem eletroestimulação } \\
\text { funcional (FES) }\end{array}$ \\
\hline $\begin{array}{l}\text { Sullivan KJ, Knowlton BJ, Dobkin BH. (ano } \\
\text { de publicação: 2002) }\end{array}$ & $\begin{array}{l}4 \text { semanas } \\
3 \text { sessões } \\
40 \% \text { de SPP progressivamente } \\
\text { diminuindo. } \\
\end{array}$ & $\begin{array}{l}\text { Grupo com velocidade de esteira lenta } \\
\text { Grupo com velocidade de esteira rápida } \\
\text { Grupo com velocidade de esteira } \\
\text { variável }\end{array}$ \\
\hline $\begin{array}{l}\text { Franceschini M, Carda S, Agosti M, } \\
\text { Antenucci R, } \\
\text { Malgrati D, Cisari C (ano de publicação: } \\
\text { 2009) }\end{array}$ & $\begin{array}{l}\text { GC } \\
5 \text { sessões/semana } \\
4 \text { semanas } \\
60 \text { minutos } \\
\text { GE } \\
5 \text { sessões/semana } \\
4 \text { semanas } \\
20 \text { minutos com SPP seguido de } 40 \\
\text { com treino convencional } \\
40 \% \text { de SPP progressivamente } \\
\text { diminuindo }\end{array}$ & $\begin{array}{l}\text { Grupo controle - treino no solo ( } 42 \\
\text { pessoas) } \\
\text { Grupo experimental - treino com SPP } \\
\text { (42 pessoas) }\end{array}$ \\
\hline $\begin{array}{l}\text { Yagura H, Hatakenaka M, Miyai I (ano } \\
\text { de publicação: 2006) }\end{array}$ & $\begin{array}{l}18 \text { sessões } \\
6 \text { semanas } \\
\text { Com } 2 \text { dias de fisioterapia extra e } \\
\text { terapia ocupacional } \\
50 \% \text { de SPP progressivamente } \\
\text { diminuindo }\end{array}$ & $\begin{array}{l}\text { Grupo Controle ( } 25 \text { indivíduos) } \\
\text { Grupo Experimental - SPP + Terapia } \\
\text { Facilitatória ( } 22 \text { indivíduos) }\end{array}$ \\
\hline
\end{tabular}

\section{DISCUSSÃO}

A partir dessa revisão sistemática, foi constatado que o SPP teve eficácia na maioria dos artigos analisados (BARBEAU e VISINTIN, 2003; BURGESS et al., 2010; DANIELSSON e SUNNERHAGEN, 2000; PEURALA et al., 2005; SULLIVAN et al., 2002; VISINTIN et al., 1998; YANG et al., 2010). Observou-se que a metodologia da maioria dos estudos ocorreu através de comparações entre grupo controle e experimental, obtendo resultados através de protocolos de tratamento fisioterapêutico convencional com uso do SPP, nos quais, a compreensão dos fatores limitantes do paciente pós-AVE foi essencial para uma melhor abordagem terapêutica, visto que qualquer intervenção deve ser direcionada a todos os aspectos que envolvem as disfunções.

O treino de marcha na esteira com SPP possibilita ao paciente praticar os parâmetros de tempo inter e intramembros antes de ter força muscular suficiente para suportar seu próprio peso corporal, permite que o carregamento do membro afetado varie de acordo com sua capacidade, força o paciente a pisar com os dois membros evitando focar somente no membro não afetado, além de prevenir o fenômeno do "não uso 
aprendido", praticar a marcha sem medo de quedas, pois fornece um senso de segurança e elimina a necessidade de movimentos adaptativos. O SPP também fornece abordagens dinâmicas e tarefas específicas que integram três componentes essenciais para marcha: equilíbrio, reforço e sustentação de peso, a esteira estimula treino intensivo que requer repetição e ritmo enquanto o paciente é sustentado na posição ortostática, o que viabiliza um treino simétrico de membros inferiores (DALY e RUFF, 2004; SHEPHERD e CARR, 1999; ZOROWITZ, 2005). Uso antecipado do SPP pode limitar a atrofia muscular e preservar a força através da carga sobre os músculos, assim como, prevenir descondicionamento e outras complicações da imobilidade, o que é o melhor meio para recuperar habilidade de marcha quando comparada a uma abordagem que enfatiza movimento e controle de músculos dos membros inferiores isolados (DANIELSSON e SUNNERHAGEN, 2000; DOBKIN, 1999, ZOROWITZ, 2005).

Apesar das numerosas vantagens do SPP na esteira, algumas desvantagens importantes devem ser consideradas. $O$ treino na esteira requer o esforço de dois ou três terapeutas para auxiliar a marcha de pacientes severamente afetados por causa da necessidade de colocar o membro parético durante a marcha e controlar os movimentos do tronco (Fig. 1) (HESSE et al., 2003). Na tentativa de solucionar a desvantagem pela necessidade de dois ou três terapeutas para auxílio da marcha foi desenvolvida a Lokomat (Fig.2) (HIDLER e WALL, 2005). É uma órtese de marcha acionada eletricamente, composta por um suporte para o quadril e duas órteses para perna criada com o objetivo de reduzir a carga de trabalho e melhorar o treino em esteira para terapeutas e pacientes, originalmente desenvolvido para pacientes com lesão de medula espinhal, oferece um ambiente mais favorável, no qual os pacientes severamente afetados possam praticar movimento de marcha a uma velocidade quase normal e esse treino pode se realizado regularmente por um longo período. Vários estudos documentaram sua eficácia (HESSE, 2006; KREWER et al., 2007; SCHMIDT et al., 2007).

É importante ressaltar que o uso do treino em esteira com SPP tem contraindicações relativas: fatores de risco para cardiopatias, história recente de trombose venosa profunda (TVP) e contraturas articulares ou artroses (HESSE et al., 2003).

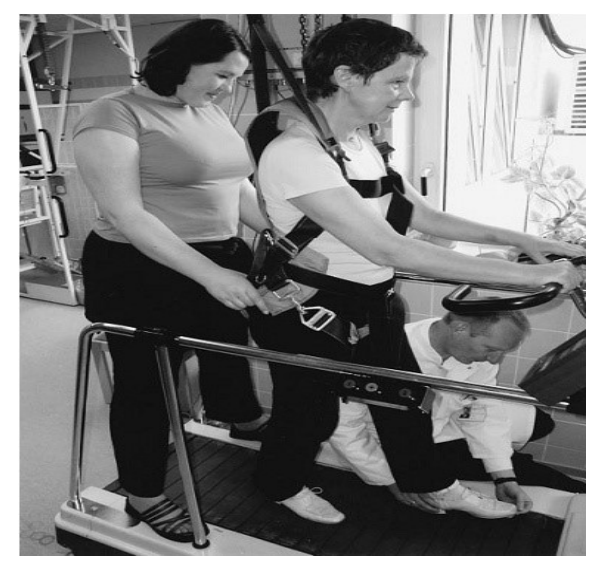

Figura 1: Paciente com hemiparesia esquerda. Treino de marcha em esteira com SPP e auxílio de dois terapeutas para posicionar seu membro.

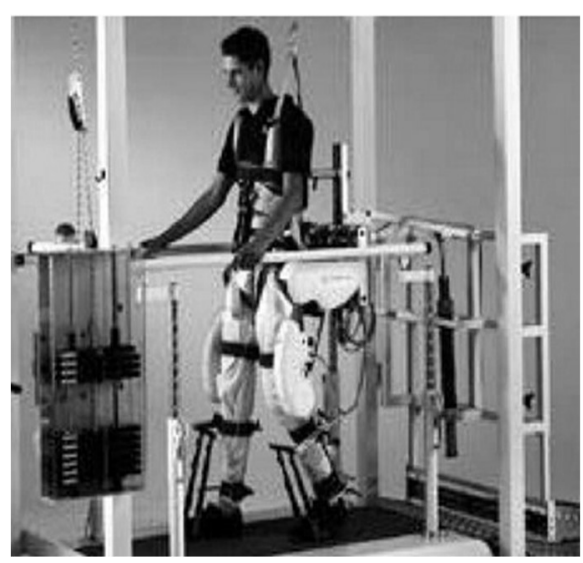

Figura 2: Paciente hemiparético com Lokomat para auxílio do treino de marcha em esteira com SPP. 


\section{CONCLUSÃO}

As evidências científicas sugerem que o SPP é eficaz no tratamento de pacientes pós AVE. No entanto, não há um consenso no protocolo para o uso deste. Considerar o estado funcional do paciente, a porcentagem de peso utilizado na hora do tratamento, o tempo de permanência do paciente na esteira e a quantidade de sessões do SPP seria fundamental para validar o protocolo e a eficiência do tratamento.

\section{REFERÊNCIAS}

AALUND, M. K.; MOE-NILSSEN, R.. Treadmill walking with body weight support Effect of treadmill, harness and body weight support systems. Gait \& Posture, v.28, p.303-308, 2008. DOI: http://dx.doi.org/10.1016/i.gaitpost.2008.01.011

BARBEAU, H.; VISINTIN, M.. Optimal outcomes obtained with body-Weight support combined with treadmill training in stroke subjects. Archives of Physical Medicine and Rehabilitation; v.84, p.1458-1465, 2003. DOI: http://dx.doi.org/10.1016/S0003-9993(03)00361-7

BARBEAU, H.; FUNG, J.; VISINTIN, M.. New Approach to Retrain Gait in Stroke and Spinal Cord Injured Subjects. Neurorehabilitation and Neural Repair, v.13, p.177, 1999.

BURGESS, J. K.; WEIBEL, G. C.; BROWN, D. A.. Overground walking speed changes when subjected to body weight support conditions for non-impaired and post stroke individuals. Journal of Neuroengineering and Rehabilitation; v.11; n.7, p.6, 2010. DOI: http://doi.org/10.1186/1743-0003-7-6

CHENA, G.; PATTENA, C.; KOTHARI, D. H.; ZAJAC, F. E.. Gait deviations associated with post stroke hemiparesis: improvement during treadmill walking using weight support, speed, support stiffness, and handrail hold. Gait \& Posture, v.22, p.57-62, 2005.

CUNHA JR., I.T.; LIM, P.A.; QURESHY, H.; HENSON, H.; MONGA, T.; PROTAS, E. J.. Gait outcomes after acute stroke rehabilitation with supported treadmill ambulation training: a randomized controlled pilot study. Archives of Physical Medicine and Rehabilitation, v.83, n.9, p.1258-1265, 2002. DOI: http://dx.doi.org/10.1053/apmr.2002.34267

DALY, J. J.; RUFF, R. L.. Feasibility of combining multi-channel functional neuromuscular stimulation with weightsupported treadmill training. Journal of the Neurological Sciences, v.255, p.105-115, 2004. DOI: http://dx.doi.org/10.1682/JRRD.2007.08.0124

DANIELSSON, A.; SUNNERHAGEN, K. S.. Oxygen consumption during treadmill walking with and without body weight support in patients with hemiparesis after stroke and in healthy subjects. Archives of Physical Medicine and Rehabilitation, v.81, p.953-957, 2000. DOI: http://dx.doi.org/10.1053/apmr.2000.6283

DAVID, D.; REGNAUX, J. P.; LEJAILLE, M.; LOUIS, A.; BUSSEL, B.; LOFASO, F.. Oxygen consumption during machineassisted and unassisted walking: a pilot study in hemiplegic and healthy humans. Archives of Physical Medicine and Rehabilitation, v.87, n.4, p.482-489, 2006. DOI: http://dx.doi.org/10.1016/j.apmr.2005.11.034
DAVIES, P.M. Weight-Supported Treadmill Training. Neurorehabilitation and Neural Repair, v.13, p.167, 1999:

DOBKIN, B. H.. An Overview of Treadmill Locomotor Training with Partial Body Weight Support: A Neurophysiologically Sound Approach Whose Time Has Come for Randomized Clinical Trials. Neurorehabilitation and Neural Repair, v.13, p.157-165, 1999. DOI: http://doi.org/10.1177/154596839901300301

FRANCESCHINI, M.; CARDS, S.; AGOSTI, M.; ANTENUCCI, R.; MALGRAT, D. E.; CESARI, C.. Walking after stroke: what does treadmill training in patients early after stroke?: a singleblind, randomized, controlled trial. Stroke, v.40, n.9, p.30793085, 2009. DOI: http://doi.org/10.1161/STROKEAHA.109.555540

FRANZ, J. R.; GLAUSER, M.; RILEY, P. O.; DELLA CROCE, U.; NEWTON, F.; ALLAIRE, P. E.; KERRIGAN, D. C.. Physiological modulation of gait variables by an active partial body weight support system. Journal of Biomechanics, v.40, n.14, p.3244-3250, 2007. DOI: http://doi.org/10.1016/j.jbiomech.2007.04.016

FUGL-MEYER, A. R.; JÄÄSKÖ, L.; LEYMAN, I.; OLSSON, S.; STEGLIND, S.. The post stroke hemiplegic patient: a method for evaluation of physical performance. Scandinavian Journal of Rehabilitation Medicine, v.7, p.13-31, 1975

HARVEY, R. L.. Motor recovery after stroke: new directions in scientific inquiry. Physical Medicine and Rehabilitation Clinics of North America, v.14, n.1, p.1-5, 2003.

HESSE, S.. Gait training after stroke: a critical reprisal. Annales de Réadaptation et de Médecine Physique, v.49, n.8, p.621-624, 2006. DOI: http://doi.org/10.1016/j.annrmp.2006.08.002

HESSE, S.; KONRAD, M.; UHLENBROCK, D.. Treadmill walking with partial body weight support versus floor walking in hemiparetic subjects. Archives of Physical Medicine and Rehabilitation, v.80, n.4, p.421-427, 1999.

HESSE, S.; WERNER, C.; VON FRANKENBERG, S.; BARDELEBEN, A.. Treadmill training with partial body weight support after stroke. Physical Medicine and Rehabilitation Clinics of North America, v.14, p.111-123, 2003.

HIDLER, J. M.; WALL, A. E.. Alterations in muscle activation patterns during robotic-assisted walking. Clinical Biomechanics, v.20, p.184-193, 2005. DOI: http://doi.org/10.1016/j.clinbiomech.2004.09.016 
KENDRICK, C.; HOLT, R.; MCGLASHAN, K.; JENNER, J. R.; KIRKER, S.. Exercising on a treadmill to improve functional mobility in chronic stroke: Case Report. Physiotherapy, v.8, n.5, p.261-265, 2001.

KOSAK, M. C.; REDING, M. J.. Comparison of Partial Body Weight-Supported Treadmill Gait Training versus Aggressive Bracing Assisted Walking Post Stroke. Neurorehabilitation e Neural Repair, v.14, p.13-19, 2000. DOI:

http://doi.org/10.1177/154596830001400102

KREWER, C.; MULLER, F.; HUSEMANN, B.; HELLER, S.; QUINTERN, J.; KOENIG, E.. The influence of different Lokomat walking conditions on the energy expenditure of hemiparetic patients and healthy subjects. Gait \& Posture, v.26, p.372-377, 2007.

LINCOLN, N.; LEADBITTER, D.. Assessment of Motor Function in Stroke Patients. Physiotherapy, v.65, n.2, p.48-51, 1979.

MAGAGNIN, V.; PORTA, A.; FUSINI, L.; LICARI, V.; BO, I.; TURIEL, M.; MOLTENI, F.; CERUTTI, S.; CAIANI, E. G. Evaluation of the autonomic response in healthy subjects during treadmill training with assistance of a robot-driven gait orthosis. Gait \& Posture, v.29, p.504-508, 2009. DOI: http://doi.org/10.1016/i.gaitpost.2008.11.013

MAGAGNIN, V.; BO, I.; TURIEL, M.; FORNARI, M.; CAIANI, E. G.; PORTA, A.. Effects of robot-driven gait orthosis treadmill training on the autonomic response rehabilitationresponsive stroke and cervical spondylotic myelopathy patients. Gait \& Posture, v.32, p.199-204, 2010.

MAGAGNIN, V.; CAIANI, E. G.; FUSINI, L.; TURIEL, M.; LICARI, V.; BO, I.; CERUTTI, S.; PORTA, A.. Assessment of the Cardiovascular Regulation During Robotic Assisted Locomotion in Normal Subjects: Autoregressive Spectral Analysis vs Empirical Mode Decomposition. .Engineering in Medicine and Biology Society Membership, v.4, p.3844$3847,2008$.

MCCAIN, K. J.; POLLO, F. E.; BAUM, B. S.; COLEMAN, S. C.; BAKER, S.; SMITH, P. S.. Locomotor treadmill training with partial body-weight support before overground gait in adults with acute stroke: a pilot study. Archives of Physical Medicine and Rehabilitation, v.89, n.4, p.684-691, 2008. DOI: http://doi.org/10.1016/j.apmr.2007.09.050

MIYAI, I.; SUZUKI, M.; HATAKENAKA, M.; KUBOTA, K.. Effect of body weight support on cortical activation during gait in patients with stroke. Experimental Brain Research, v.169, p.85-91, 2006.

MOSELEY, A.M.; STARK, A.; CAMERON, I.S.; POLLOCK, A.. Treadmill training and body weight support for walking after stroke. The Cochrane Database of Systematic Review, v.19, n.4, 2005. DOI: http://doi.org/10.1002/14651858.CD002840

NILSSON, L.; CARLSSON, J.; DANIELSSON, A.. Walking training of patients with hemiparesis at an early stage after stroke: a comparison of walking training on a treadmill with body weight support and walking training on the ground. Clinical Rehabilitation, v.15, p.515-527, 2001. DOI: http://doi.org/10.1191/026921501680425234

PEURALA, S. H.; TARKKA, I. M.; PITKÄNEN, K.; SIVENIUS, J.. The effectiveness of body weight-supported gait training and floor walking in patients with chronic stroke. Archives of Physical Medicine and Rehabilitation, v.86, p.1557-1564, 2005. DOI: http://doi.org/10.1016/j.apmr.2005.02.005

RAMAS, J.; COURBONA, A.; ROCHE, F.; BETHOUXC, F.; CALMELS, $P$.. Effect of training programs and exercise in adult stroke patients: literature review. Annales de Réadaptation et de Médecine Physique, v.50, p.438-444, 2007. DOI: http://doi.org/10.1016/j.annrmp.2007.04.006

REGNAUX, J. P.; SAREMI, K.; MAREHBIAN, J.; BUSSEL, B.; DOBKIN, B. H.. An Accelerometry-Based Comparison of 2 Robotic Assistive Devices for Treadmill Training of Gait. Neurorehabilitation and Neural Repair, v.22, n.4, p.348354, 2008. DOI: http://doi.org/10.1177/1545968307310050

SABARI, J. S.; LIM, A. L.; VELOZO, C. A.; LEHMAN, L.; KIERAN, O.; LAI, J. S.. Assessing Arm and Hand Function After Stroke: A Validity Test of the Hierarchical Scoring System Used in the Motor Assessment Scale for Stroke. Archives of Physical Medicine and Rehabilitation, v.86, n.8, p.1609-1615, 2005. DOI: http://dx.doi.org/10.1016/j.apmr.2004.12.028

SAMPAIO, R. F.; MANCINI, M. C.. Estudos de revisão sistemática: um guia para a síntese criteriosa da evidência científica. Revista Brasileira de Fisioterapia, São Carlos, v.11, n.1, p. 83-89, 2007.

SCHMIDT, H.; WERNER, C.; BERNHARDT, R.; HESSE, S.; KRÜGER, J.. Gait rehabilitation machines based on programmable footplates. Journal of Neuroengineering and Rehabilitation, v.9, n.4, p.2, 2007. DOI: http://doi.org/10.1186/1743-0003-4-2

SHEPHERD, R.; CARR, J.. Treadmill Walking in Neurorehabilitation. Neurorehabilitation Neural Repair, v.13, p.171, 1999.

SOUSA, C. O.; BARELA, J. A.; MEDEIROS, C. L. P.; SALVINI, T. F.; BARELA, A. N. D.. The use of body weight support on ground level: an alternative strategy for gait training of individuals with stroke. Journal of Neuroengineering and Rehabilitationv, v.6, p.43, 2009.

SULLIVAN, K. J.; KNOWLTON, B. J.; DOBKIN, B. H.. Step training with body weight support: effect of treadmill speed and practice paradigms on post stroke locomotor recovery. Archives of Physical Medicine and Rehabilitation, v.83, p.683-691, 2002

THRELKELD, A. J.; COOPER, L. D.; MONGER, B. P.; CRAVEN, A. N.; HAUPT, H. G.. Temporospatial and kinematic gait alterations during treadmill walking with body weight suspension. Gait \& Posture, v.17, n.3, p.235-245, 2003.

VISINTIN, M.; BARBEAU, H.; KORNER-BITENSKY, N.; MAYO, N. E.. A new approach to retrain gait in stroke patients through body weight support and treadmill stimulation. Stroke, v.29, p. 1122-1128, 1998.

WALKER, M. L.; RINGLEB, S. I.; MAIHAFER, C. G.; WALKER, R.; CROUCH, J. R.; LUNEN, B. V.; MORRISON, S.. Virtual RealityEnhanced Partial Body Weight-Supported Treadmill Training Post stroke: Feasibility and Effectiveness in 6 Subjects. Archives of Physical Medicine and Rehabilitation, v.91, p.115-122, 2010. 
WERNER, C.; VON FRANKENBERG, S.; TREIG, T.; KONRAD, M.; HESSE, S.. Treadmill Training With Partial Body Weight Support and an Electromechanical Gait Trainer for Restoration of Gait in Subacute Stroke Patients: A Randomized Crossover Study. Stroke, v.33, p.2895-2901, 2002.

YAGURA, H.; HATAKENAKA, M.; MIYAI, I.. Does therapeutic facilitation add to locomotor outcome of body weightsupported treadmill training in nonambulatory patients with stroke? A randomized controlled trial. Archives of Physical Medicine and Rehabilitation, v.87, p. 529-535, 2006. DOI: http://doi.org/10.1016/j.apmr.2005.11.035
YANG, Y. R.; CHEN, I. H.; LIAO, K. K.; HUANG, C. C.; WANG, R. $\mathrm{Y}$.. Cortical reorganization induced by body weightsupported treadmill training in patients with hemiparesis of different stroke durations. Archives of Physical Medicine and Rehabilitation, v.91, p.513-518, 2010. DOI: http://doi.org/10.1016/i.apmr.2009.11.021

ZOROWITZ, R. D.. Ambulation in a Wheelchair-Bound Stroke Survivor Using a Walker with Body Weight Support: A Case Report. Topics in Stroke Rehabilitation, v.12, n.4, p.50-55, 2005. DOI: http://doi.org/10.1310/7JYP-2NYH-5QQQ-5FFR 